\title{
Preoperative arterial embolization of heterotopic ossification around the hip joint
}

\author{
Jin Hyeok Kim¹, Chankue Park ${ }^{1}$, Seung Min Son ${ }^{2}$, Won Chul Shin ${ }^{2}$, Joo Yeon Jang ${ }^{1}$, \\ Hee Seok Jeong', In Sook Lee ${ }^{3}$, Tae Young Moon ${ }^{1}$ \\ Departments of ${ }^{1}$ Radiology and ${ }^{2}$ Orthopedic Surgery, Pusan National University Yangsan Hospital, Yangsan; \\ ${ }^{3}$ Department of Radiology, Pusan National University Hospital, Busan, Korea
}

\begin{abstract}
Heterotopic ossification $(\mathrm{HO})$ around the hip joint is not uncommon following neurological injury. Often, surgical treatment is performed in patients with restricted motion and/or refractory pain due to grade III or IV $\mathrm{HO}$ according to Brooker classification. The major complication that occurs as a result of surgical $\mathrm{HO}$ removal is perioperative bleeding due to hyper-vascularization of the lesion. Here, we report a case of preoperative embolization in a 51-year-old male patient presenting with restricted bilateral hip range of motion (ROM) due to $\mathrm{HO}$ following a spinal cord injury. In the right hip without preoperative arterial embolization, massive bleeding occurred during surgical removal of $\mathrm{HO}$. Thus, the patient received a transfusion postoperatively due to decreased serum hemoglobin levels. For surgery of the left hip, preoperative embolization of the arteries supplying $\mathrm{HO}$ was performed. Surgical treatment was completed without bleeding complications, and the patient recovered without a postoperative transfusion. This case highlights that, while completing surgical removal for ROM improvements, orthopedic surgeons should consider preoperative arterial embolization in patients with hip HO.
\end{abstract}

Keywords: Hip; Heterotopic ossification; Arterial embolization; Gelatin sponge

\section{INTRODUCTION}

Heterotopic ossification $(\mathrm{HO})$ is defined as the abnormal formation of mature trabecular bone in extraskeletal soft tissues. It is not uncommon complication following trauma, surgery, neurological injury, and severe burns [1,2]. The hip is the most common site of $\mathrm{HO}$, followed by the elbow, knee, and shoulder [3,4]. HO manifests in various manners, from islands of the bone within the soft tissues about the hip, to

Received: April 18, 2018, Revised: May 18, 2018 Accepted: May 21, 2018

Corresponding Author: Chankue Park, Department of Radiology, Pusan National University Yangsan Hospital,

20, Geumo-ro, Mulgeum-eup, Yangsan 50612, Korea

Tel: +82-55-360-1840, Fax: +82-55-360-1848

E-mail: chankue.park@gmail.com apparent hip bone ankylosis. Although small foci of $\mathrm{HO}$ are not clinically significant, surgical treatment is sometimes required in patients presenting with restricted motion or refractory pain due to extensive $\mathrm{HO}$ [5]. Surgical excision of $\mathrm{HO}$ is challenging to surgeons for several important reasons. Proper timing of surgical intervention must be considered, excision is a difficult technique, and $\mathrm{HO}$ has a possibility of recurrence. Furthermore, due to hypervascularization of $\mathrm{HO}$ and adhesion between the $\mathrm{HO}$ and the surrounding soft tissues, intra- and post-operative bleeding are major potential complications [6,7].

We report a case of preoperative arterial embolization of $\mathrm{HO}$ in a patient presenting with restricted bilateral hip range of motion (ROM). This case highlights that preoperative arterial embolization is useful to reduce intra- and post-operative bleeding complications if patients with hip joint $\mathrm{HO}$ require

Copyright $($ C) 2018 Yeungnam University College of Medicine

This is an Open Access article distributed under the terms of the Creative Commons Attribution Non-Commercial License (http://creativecommons.org/licenses/by-nc/4.0/) which permits unrestricted non-commercial use, distribution, and reproduction in any medium, provided the original work is properly cited. 
HO removal for improvements in their ROM.

\section{CASE}

The patient and his family were informed that this case would be submitted for publication. A 51-year-old man visited the hospital for progressively restricted hip motion. He experienced difficulties achieving proper seated posture due to severe restricted ROM of the hip, and presented with severe hip pain. He was in a paraplegic state due to T11 cord injury following a fall 10 months ago. On physical examination, he showed $30^{\circ}$ of flexion contracture in both hip joints, and
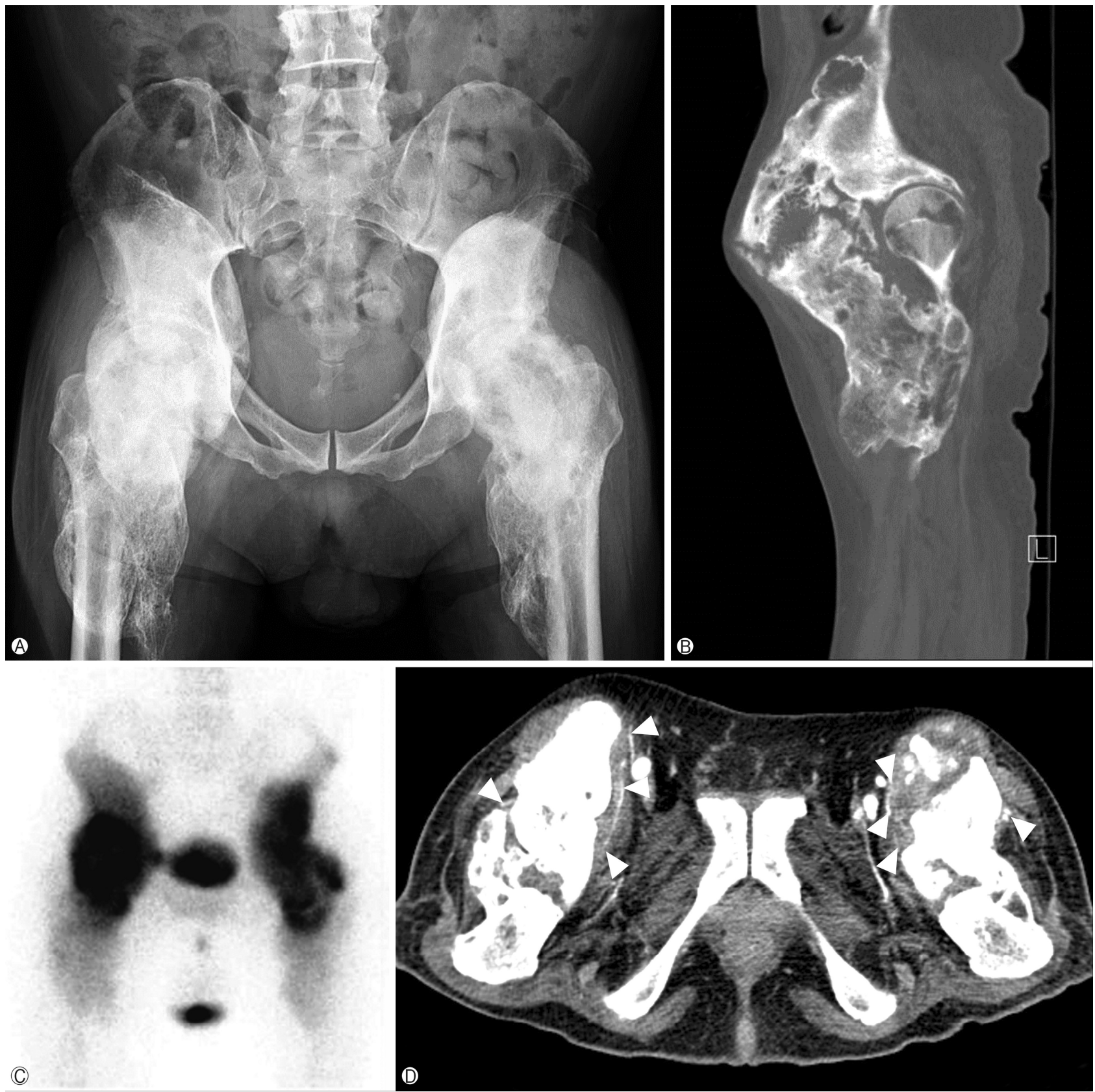

Fig. 1. (A) Pelvis anteroposterior radiograph shows diffuse heterotopic ossification around both hip joints. (B) Sagittal reconstruction computed tomography (CT) image shows nearly complete bone ankylosis of the left hip joint, causing total stiffness of the hip joint. (C) Technetium 99m-methyl diphosphonate bone scan shows intense uptake in bilateral hip joints. (D) Preoperative axial CT with contrast enhancement shows hypertrophied arteries (arrowheads) supplying the heterotopic bone formation around the bilateral hip joints. 
no further degree of ROM. The patient underwent radiologic and laboratory evaluations. Pelvic anteroposterior radiography and hip computed tomography (CT) showed massive heterotopic bone formation around bilateral hip joints and apparent bone ankylosis of the joints (Fig. 1A, 1B). Scintigraphic bone scan showed increased uptake in bilateral hip joints, suggesting active lesion (Fig. 1C). Serum alkaline phosphatase (ALP) levels were measured as $329 \mathrm{IU} / \mathrm{L}$ (range, 30-120 IU/L). Serum
ALP levels were measured during follow-up outpatient appointments until the $\mathrm{HO}$ matured. During the 1-year follow up, ALP levels fell within the normal range, and the patient decided to undergo surgery for persistent hip contracture and limited ambulation necessitating wheelchair usage. Preoperative CT with contrast enhancement showed heterotopic bone formation in both hip areas. Hypertrophied bilateral superior gluteal and lateral circumflex arteries supplying the hetero-
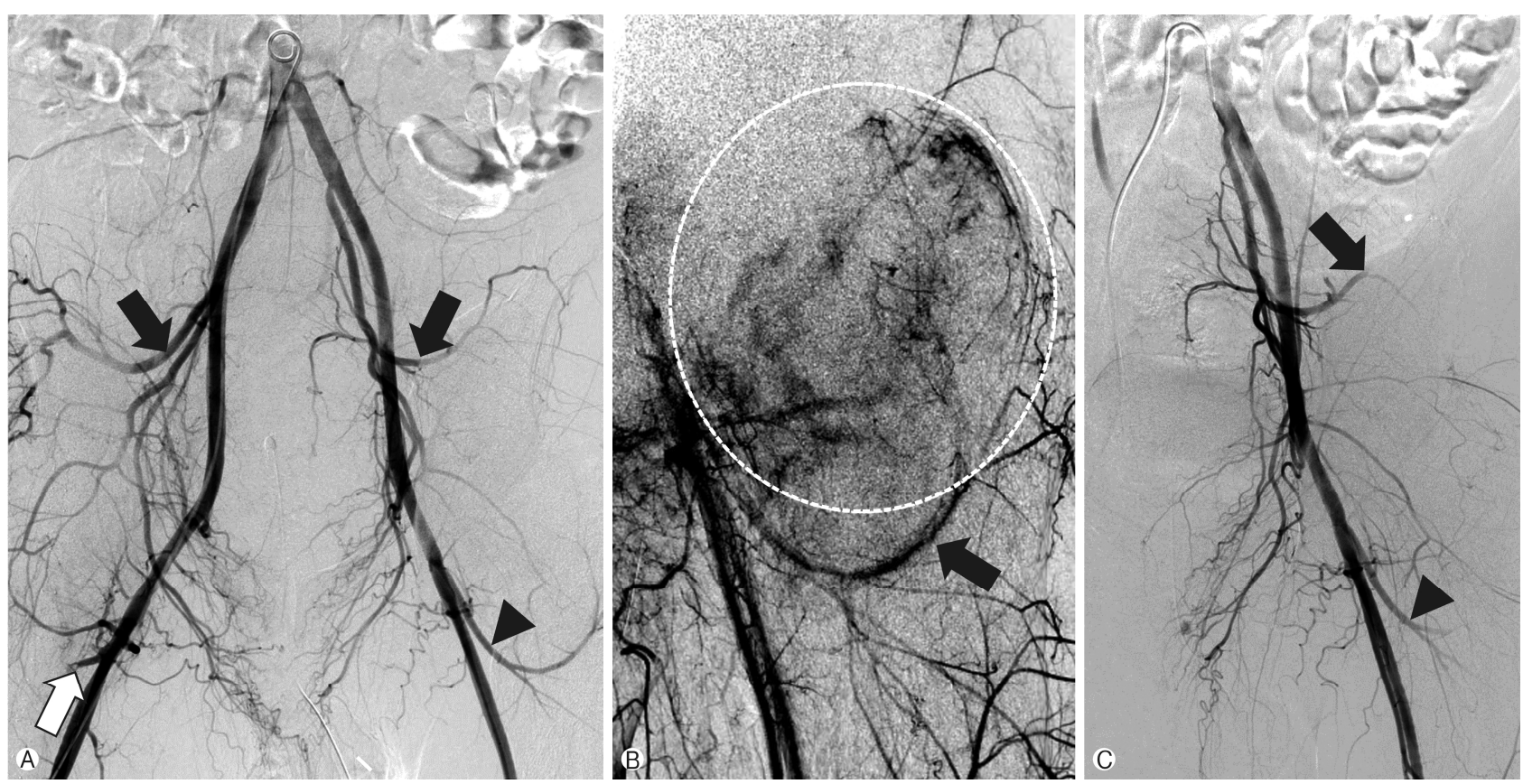

Fig. 2. (A) Common iliac arteriogram reveals hypertrophied bilateral superior gluteal arteries (black arrows), left lateral circumflex femoral artery (arrowhead), and the stump of right lateral circumflex femoral artery (white arrow), which was ligated during the previous surgery. (B) External iliac angiography demonstrates hypertrophied left lateral circumflex femoral artery (arrow) and diffuse hypervascular area involving the left hip joint (dashed circle). Note that the left lateral circumflex femoral artery arises from common femoral artery, as a normal anatomical variant. (C) Angiography obtained after embolization with gelatin sponge slurry shows decreased vascular flow in the left superior gluteal artery (arrow) and lateral circumflex femoral artery (arrowhead).
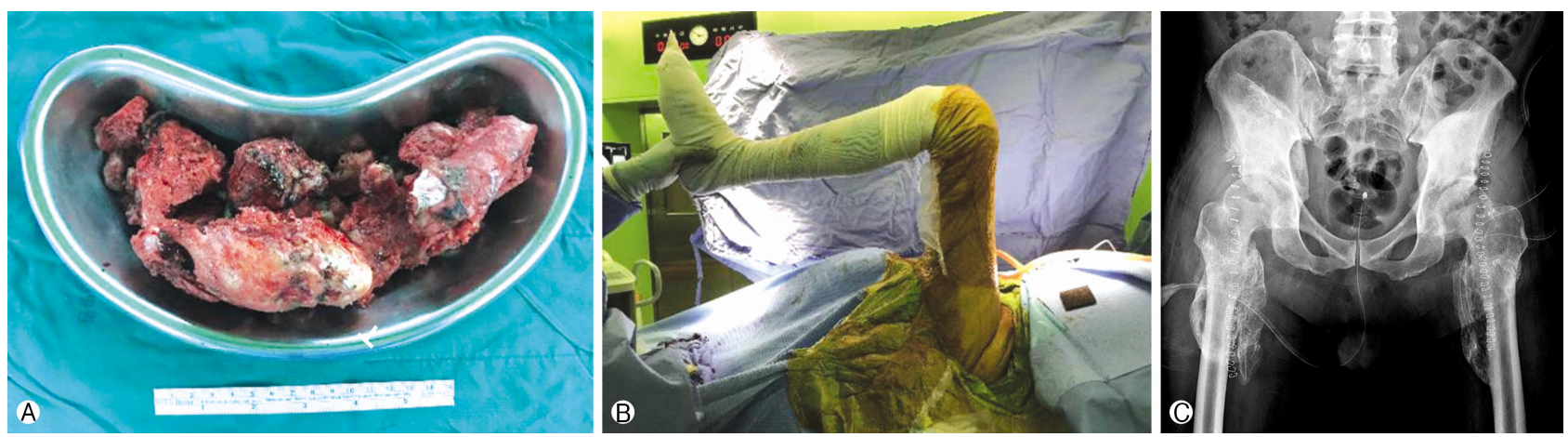

Fig. 3. (A) Gross specimen showing multiple bone fragments with hemorrhage. (B) Passive flexion of the left hip joint was available after surgical removal of heterotopic ossification. (C) Immediate postoperative pelvis anteroposterior radiograph shows a decreased extent of heterotopic ossification compared with that in the preoperative pelvic radiograph. 
topic bone formation around the bilateral hip joints were also visualized (Fig. 1D). The surgeon planned a two-step surgery for heterotopic bone formation removal: the right side first, followed by the left side.

In the surgery around the right hip joint, it was difficult to detect the right lateral femoral circumflex artery due to bleeding and severe adhesion between the heterotopic bone formation and the surrounding soft tissue. Rupture of the right lateral circumflex femoral artery and subsequent massive bleeding occurred. Ligation of the ruptured right lateral circumflex artery was performed, and surgery was finished after partial resection of the soft tissue bone formation. A preoperative hemoglobin concentration of $14.4 \mathrm{~g} / \mathrm{dL}$ (hematocrit $40.4 \%$ ) decreased to $10.7 \mathrm{~g} / \mathrm{dL}$ (hematocrit $30.6 \%$ ) on postoperative day $1,9.5 \mathrm{~g} / \mathrm{dL}$ (hematocrit $27.6 \%$ ) on postoperative day 2 , and $8.3 \mathrm{~g} / \mathrm{dL}$ (hematocrit 23.9\%) on postoperative day 3. Two units of whole blood were administered to the patient, and the value increased to $11.0 \mathrm{~g} / \mathrm{dL}$ (hematocrit $32.0 \%$ ) on postoperative day 7 .

Contrary to the right hip surgery, preoperative arterial embolization around the left hip joint was planned to prevent perioperative blood loss. Bilateral common iliac angiography, performed by puncturing the right femoral artery using the Seldinger method (a standard transfemoral approach) showed hypertrophied bilateral superior gluteal arteries, a hypertrophied left lateral circumflex femoral artery, and a stump of the ligated right lateral circumflex femoral artery (Fig. 2A). On selective left external iliac angiography, a hypertrophied left lateral circumflex femoral artery and diffuse hypervascular area involving the left hip joint area were observed (Fig. 2B). After selection of the left lateral circumflex femoral artery and left superior gluteal artery using a microcatheter (Renegade, Boston Scientific, Natick, MA, USA), embolization was performed with a gelatin sponge called a gelfoam (Cutanplast, CureSys, Seoul, Korea). The gelatin sponge slurry was created by forcefully mixing small gelatin sponge particles with a solution of contrast material. Postembolization external iliac angiography revealed decreased vascular flow in the left lateral circumflex femoral artery and superior gluteal artery supplying the heterotopic bone formation around the left hip joint (Fig. 2C). The patient was observed 6 hours post-intervention and did not have any complications. On the next day, surgical removal of the heterotopic bone formation was successfully performed without massive bleeding and ended after confirmation of 100 degrees of left hip joint flexion by checking the intraoperative hip ROM (Fig. 3). Total surgery time of the right and left hips was 2 hours 40 minutes, and 2 hours, respectively. Blood loss of the right and left hip was $1,500 \mathrm{~mL}$ and $500 \mathrm{~mL}$, respectively. Intraoperative transfusion was not performed in either right or left hip surgeries. On postoperative day 3 of the left hip, the preoperative hemoglobin value of $11.0 \mathrm{~g} / \mathrm{dL}$ (hematocrit 32.0\%) reached a minimum value of $9.3 \mathrm{~g} / \mathrm{dL}$ (hematocrit $27.4 \%$ ), but recovered to $10.6 \mathrm{~g} / \mathrm{dL}$ (hematocrit 33.0\%) without transfusion. Pathology confirmed bone formation around both hip joints as HO. A drainage catheter in the operated site was removed, and the patient began rehabilitation exercises, including continuous passive motion, on postoperative day 1 . He was administered single radiation therapy of $800 \mathrm{~Gy}$ to the operated site, and started to take celecoxib $200 \mathrm{mg}$ orally one time per day for a total of 6 weeks for prophylaxis of $\mathrm{HO}$ recurrence. He has been able to ambulate in a wheelchair without pain after surgery, and $\mathrm{HO}$ has yet to recur.

\section{DISCUSSION}

The hip region is the most common site of $\mathrm{HO}$, a common complication (0-44\% without prophylaxis) after hip arthroscopy $[3,4]$. Although HO is usually asymptomatic, grade III or IV $\mathrm{HO}$ of Brooker classification around a joint significantly limits joint function, and may require a surgical treatment $[5,8,9]$.

$\mathrm{HO}$ is initiated by local tissue damage, leading to increased bone morphogenic protein 2 targeting of the sensory nerves [10]. Subsequently, a cascade of molecular mechanisms induces peripheral nerve remodeling [7]. The production of chondro-osseous, glial, vascular, and neural progenitor cells following the remolding induces vascularization, and increases levels of vascular endothelial growth factor that promotes vessel formation [6,7]. Due to the increased vascularization, bleeding is a major peri- and post-operative complication.

Preoperative transcatheter arterial embolization of hypervascular lesions is commonly performed to decrease blood loss during surgery, improve visualization of the surgical field, and shorten operative time [11,12]. Embolic materials are selected according to target vessel size and occlusion duration. A gelatin sponge is the most widely used absorptive temporary embolic agent, typically lasting 3-6 weeks of vascular 
occlusion. It is inexpensive, easy to use with a microcatheter, and can be modified depending on target vessel size [12,13]. By performing preoperative embolization with a gelatin sponge slurry, ischemia to other areas that are supplied by the same feeding arteries of the $\mathrm{HO}$ can be minimized by expecting recanalization of the occluded vessel after surgery.

Preoperative arterial embolization using a gelatin sponge may be useful for surgical removal of extensive $\mathrm{HO}$ in the hip joint area. Its purpose is to decrease blood loss, improve visualization of surgical field, and shorten operative time.

\section{CONFLICT OF INTEREST}

No potential conflict of interest relevant to this article was reported.

\section{ORCID}

Jin Hyeok Kim, https://orcid.org/0000-0001-6703-2419

Chankue Park, https://orcid.org/0000-0003-2937-114X

\section{REFERENCES}

1. Clements NC Jr, Camilli AE. Heterotopic ossification complicating critical illness. Chest 1993;104:1526-8.

2. Hannallah D, Peng H, Young B, Usas A, Gearhart B, Huard J. Retroviral delivery of Noggin inhibits the formation of heterotopic ossification induced by BMP-4, demineralized bone matrix, and trauma in an animal model. J Bone Joint Surg Am 2004;86:80-91.

3. Almangour W, Schnitzler A, Salga M, Debaud C, Denormandie $\mathrm{P}$, Genêt F. Recurrence of heterotopic ossification after removal in patients with traumatic brain injury: a systematic review. Ann Phys Rehabil Med 2016;59:263-9.

4. Bedi A, Zbeda RM, Bueno VF, Downie B, Dolan M, Kelly BT. The incidence of heterotopic ossification after hip arthroscopy. Am J Sports Med 2012;40:854-63.

5. Board TN, Karva A, Board RE, Gambhir AK, Porter ML. The prophylaxis and treatment of heterotopic ossification following lower limb arthroplasty. J Bone Joint Surg Br 2007; 89:434-40.

6. Dilling CF, Wada AM, Lazard ZW, Salisbury EA, Gannon $\mathrm{FH}$, Vadakkan TJ, et al. Vessel formation is induced prior to the appearance of cartilage in BMP-2-mediated heterotopic ossification. J Bone Miner Res 2010;25:1147-56.

7. Reichel LM, Salisbury E, Moustoukas MJ, Davis AR, OlmstedDavis E. Molecular mechanisms of heterotopic ossification. J Hand Surg Am 2014;39:563-6.

8. Warren SB, Brooker AF Jr. Intramedullary nailing of tibial nonunions. Clin Orthop Relat Res 1992;285:236-43.

9. Baird EO, Kang QK. Prophylaxis of heterotopic ossification - an updated review. J Orthop Surg Res 2009;4:12.

10. Salisbury E, Rodenberg E, Sonnet C, Hipp J, Gannon FH, Vadakkan TJ, et al. Sensory nerve induced inflammation contributes to heterotopic ossification. J Cell Biochem 2011;112: 2748-58.

11. Rilling WS, Chen GW. Preoperative embolization. Semin Intervent Radiol 2004;21:3-9.

12. Vogl TJ, Wolff JD, Balzer J, Skripitz R. Preoperative arterial embolization in heterotopic ossification: a case report. Eur Radiol 2001;11:962-4.

13. Medsinge A, Zajko A, Orons P, Amesur N, Santos E. A casebased approach to common embolization agents used in vascular interventional radiology. AJR Am J Roentgenol 2014; 203:699-708. 